\title{
Ninjurin1 mediates macrophage-induced programmed cell death during early ocular development
}

\author{
H-J Lee ${ }^{1}$, BJ Ahn ${ }^{1}$, MW Shin ${ }^{1}$, J-W Jeong ${ }^{2}$, JH Kim ${ }^{3}$ and K-W Kim ${ }^{\star, 4}$
}

Developmental tissues go through regression, remodeling, and apoptosis. In these processes, macrophages phagocytize dead cells and induce apoptosis directly. In hyaloid vascular system (HVS), macrophages induce apoptosis of vascular endothelial cells (VECs) by cooperation between the Wnt and angiopoietin (Ang) pathways through cell-cell interaction. However, it remains unclear how macrophages are activated and interact with VECs. Here we show that Ninjurin1 (nerve injury-induced protein; Ninj1) was temporally increased in macrophages during regression of HVS and these Ninj1-expressing macrophages closely interacted with hyaloid VECs. Systemic neutralization using an anti-Ninj1 antibody resulted in the delay of HVS regression in vivo. We also found that Ninj1 increased cell-cell and cell-matrix adhesion of macrophages. Furthermore, Ninj1 stimulated the expression of Wnt7b in macrophages and the conditioned media from Ninj1-overexpressing macrophages (Ninj1-CM) decreased Ang1 and increased Ang2 in pericytes, which consequently switched hyaloid VEC fate from survival to death. Collectively, these findings suggest that macrophages express Ninj1 to increase the death signal through cell-cell interaction and raise the possibility that Ninj1 may act similarly in other developmental regression mediated by macrophages.

Cell Death and Differentiation (2009) 16, 1395-1407; doi:10.1038/cdd.2009.78; published online 26 June 2009

Developmental tissues including embryonic tail, ${ }^{1}$ interdigital epithelium, ${ }^{2}$ neuron, ${ }^{3}$ and $\mathrm{HVS}^{4}$ undergo regression, remodeling, and programmed cell death. During these processes, many cells die by apoptosis or phagocytosis and are eliminated. Macrophages exist in all tissues of the body under normal conditions and the number of these cells increases markedly with the onset and progression of developmental regression as well as many pathological diseases. ${ }^{1-3}$ In addition, activated macrophages can move and attach to the target cells producing cytokines and signaling molecules. Such activation is strongly related with the regression of the HVS..$^{5-7}$

HVS is a transient existing network of capillaries and consists of the hyaloid artery (HA), vasa hyaloidea propria (VHP), tunica vasculosa lentis (TVL), and pupillary membrane (PM). This vasculature is important for the growth and maturation of lens, because it supplies oxygen and nutrients to the developing lens. Moreover, HVS regresses contemporaneously with the formation of retinal vasculature, suggesting its involvement in the formation of the primary vascular vitreous of the developing eye. ${ }^{4}$ Therefore, abnormal regression or proliferation of the vasculature is associated with congenital ocular pathologies such as persistent hyperplastic tunica vasculosa lentis (PHTVL), persistent hyperplastic primary vitreous (PHPV), and persistent prepupillary membrane (PPM). ${ }^{8}$
There are three major explanations contributing to HVS regression during ocular development. First, macrophages reportedly induce programmed cell death and drive the regression of the HVS using ablation experiments. ${ }^{9-11}$ For example, mice deficient in lymphomyeloid transcription factor PU.1, which lack macrophages, appeared to persist HVS postnatally. ${ }^{7}$ There are some molecules related to the activation of macrophages during HVS regression such as Wnt7b and BMP-4. ${ }^{12}$ Especially, Wnt7b is reported as a critical mediator of cell death in the HVS. It activates the Wnt pathway in adjacent VECs, most likely through direct cell-cell contact, because Wnt7b may be highly insoluble after posttranslational modification in the endoplasmic reticulum (ER) by palmitate attachment and the lipid-modified Wnt7b is linked to plasma membrane. ${ }^{7,13}$ This Wnt7b induces cell cycle of VECs, leading to increased sensitivity of VECs to cell death. Secondly, angiopoietin (Ang) signaling system is related to HVS regression. Angiopoietin receptor is tyrosine kinase receptor Tie2 and its best-characterized ligands are Ang1 and Ang2. Ang1 is an agonist and is associated with cell survival through the PI3-kinase-Akt signaling pathway. In contrast to Ang1, Ang2 is an antagonist and blocks the effect of Ang1, leading to suppression of survival signal and destabilizing vessels. In HVS, pericytes produce Ang2, which inhibits the

\footnotetext{
${ }^{1}$ NeuroVascular Coordination Research Center, College of Pharmacy and Research Institute of Pharmaceutical Sciences, Seoul National University, Seoul 151-742, Korea; ${ }^{2}$ Deparment of Anatomy and Neurobiology, Biomedical Science Institute, School of Medicine, Kyung Hee University, Seoul 130-701, Korea; ${ }^{3}$ Department of Ophthalmology, Seoul National University College of Medicine and Seoul Artificial Eye Center, Clinical Research Institute, Seoul National University Hospital, Seoul 110744, Korea and ${ }^{4}$ NeuroVascular Coordination Research Center, College of Pharmacy and Department of Molecular Medicine and Biopharmaceutical Sciences, Graduate School of Convergence Science and Technology, Seoul National University, Seoul 151-742, Korea

${ }^{*}$ Corresponding author: K-W Kim, NeuroVascular Coordination Research Center, College of Pharmacy and Department of Molecular Medicine and Biopharmaceutical Sciences, Graduate School of Convergence Science and Technology, Seoul National University, Seoul 151-742, Republic of Korea. Tel: +8228806988 ;

Fax: + 822885 1827; E-mail: qwonkim@plaza.snu.ac.kr

Keywords: vascular biology; apoptosis; cell adhesion; Ninjurin1; macrophage

Abbreviations: Ang1/2, angiopoietin-1/2; col. I/IV, type I/IV collagen; CM, conditioned media; ECM, extracellular matrix; FN, fibronectin; $h$, hour; HVS, hyaloid vascular system; MMP1, matrix metalloproteinase 1; Ninjurin1 (Ninj1), nerve injury-induced protein 1; ONH, optic nerve head; P, postnatal day; R, retina; RV, retinal vessel; V, vitreous; VEC, vascular endothelial cell; VEGF, vascular endothelial growth factor; Wnt7b, wingless-type MMTV integration site 7B from Drosophila melanogaster Received 03.2.09; revised 12.5.09; accepted 20.5.09; Edited by M Piacentini; published online 26.6.09
} 
PI3-kinase-Akt (survival signal) in VECs and also promotes Wnt7b expression in macrophages; this in turn leads to apoptosis of VECs. ${ }^{6,7}$ The third explanation is that HVS regression is induced by a loss of survival factor, vascular endothelial growth factor (VEGF). Absence or decrease of VEGF expression may induce apoptosis of the VECs ${ }^{14}$ and overexpression of VEGF in the lens increased the accumulation of angioblast and VECs, indicating that VEGF is important for stability and maturation of HVS. ${ }^{15}$ However, there are some contradictory reports that VEGF expression is enhanced rather than decreased in the lens during HVS regression and remains in the adult. ${ }^{16,17}$ Thus, although VEGF is crucial for VECs stability, apoptosis of VEC may be independent on VEGF during HVS regression. Altogether, it has been accepted that cell-cell interaction between macrophages, endothelial cells, and pericytes is crucial for the HVS regression. However, it remains largely unknown how macrophages are activated and interact closely with VECs and how Wnt-Ang pathway is activated during HVS regression.

Here we found that macrophages transiently expressed Ninj 1 to migrate and adhere to the VECs and activate Wnt7bAng pathway leading to HVS regression.

Ninjurin family proteins (Ninjurins) are multipass membrane proteins induced by nerve injury, increasing cell adhesion, and promoting axonal growth in Schwann cells and dorsal root ganglion neurons. ${ }^{18,19}$ In the mammal, there are two different Ninjurins, Ninj1 and Ninj2, which share conserved hydrophobic regions for their transmembrane domains; however, they differ in their adhesion motifs and cellular expression patterns. ${ }^{19}$ The amino-acid sequence of the human Ninj1 is $55 \%$ identical to human Ninj2 but has no significant homology to any other known proteins. ${ }^{19}$ Furthermore, the tissue distribution of Ninjurins shows significant difference. In addition to injured nervous system, Ninj 1 is widely expressed in adult and embryonic tissues, particularly those with epithelial origin. ${ }^{18,19}$ Meanwhile, Ninj2 is more restrictedly expressed, with highest levels in adult bone marrow and embryonic thymus. ${ }^{20}$ It is important that Ninj1 is expressed in a number of other tissues, predominantly in epithelial cells, suggesting that Ninj1 may be involved in multiple functional systems in the body and also play a role in the formation and function of other tissues such as neuronal development as well as nerve regeneration like other known adhesion molecules (N-CAM, or L1). ${ }^{18,21-23}$ Recently, it has been reported that Drosophila NijA (a fly homolog of vertebrate Ninj1) regulates the remodeling of the tracheal system through interaction with matrix metalloproteinase 1 (MMP1), suggesting that it may be involved in the process of vascular remodeling in higher vertebrates. ${ }^{24}$ In this study, we showed that transiently expressed Ninj1 increases cell-cell and cell-matrix adhesion of macrophages and enhances apoptosis of hyaloid VEC through the Wnt7b-Ang pathway, leading to HVS regression. Collectively, we demonstrate that Ninj1 has an important role in vascular regression mediated by macrophages during early eye development and suggest the biological relevance of Ninj1 on vascular homeostasis.

\section{Results}

The spatiotemporal expression of Ninj1 during HVS regression. In flat-mounted ocular specimens of rodents, GS-lectin (a macrophage and endothelial cell marker) staining clearly showed that HVS regressed, with characteristic narrowing and thinning in vascular diameter along with decrease of interconnecting vessels (Figure 1a). In Supplementary Figure S1, white-clover images correspond to their original images of HVS vasculature. This clearly illustrated that vascular density decreases extensively from postnatal day 4 (P4) to P8. Although the HVS nearly atrophied around $\mathrm{P} 10$, vascular plexus grew from the optic nerve head $(\mathrm{ONH})$ into the retina (Figure 1a). Interestingly, we found that Ninj1 was detectable from P1 through P14, with a peak of expression at P5 (Figure 1b), whereas it was not detectable in adult eyes (data not shown). In contrast, the vascular density of HVS decreased consistently during the same period, especially from P3 to P8 (Figure 1c). This is consistent with the notion that the greatest changes in hyaloid vasculature occur between P4 and P5, especially in VHP branches of HVS; ${ }^{25}$ therefore, these data suggest that transiently expressed Ninj1 during early retinal development may play a role in the regression of HVS.

To examine the spatiotemporal pattern of Ninj1 immunoreactivity during HVS regression in vivo, we immunostained the cryosectioned or flat-mounted P1-P14 ocular specimens. Round-shaped and ovoid cells were stained with anti-Ninj1 antibodies in the vitreous, with the highest expression observed at P5 (Figure 1d). These Ninj1-positive cells were linearly arranged around the vessel-like structures, which were located in the vitreous and around the lens, but not in the retina (Figure 1e). As Ninj1-positive cells appeared near the vessels, we performed a double staining with VE-cadherin (an endothelial marker) to confirm these observations. Ninj1 staining did not colocalize with VE-cadherin signal; instead, Ninj1 signal was detected around VE-cadherin-positive cells, suggesting that Ninj1-positive cells were located near the HVS (Figure 1f). These data indicate that Ninj1 might be expressed exclusively in round-shaped cells in the vitreous around the HVS.

Figure 1 The temporal and spatial expression of Ninj1 during ocular development. (a) GS-lectin (green) staining showed HVS and primary retinal vessels in P2-P14 and adult whole-mounted retinas. White dotted lines indicate retinal vasculature growth. HV: hyaloids vasculature; RV: retinal vasculature; ONH: optic nerve head. (b) Western blotting showed an increase of Ninj1 protein in P3-P5 rat eyes when compared with fully regressed P14 eyes. An $\alpha$-tubulin antibody was used as an internal control. (c) Quantification of vascular density $(\boldsymbol{\Lambda})$ and expression of Ninj1 $(\boldsymbol{O})$ during ocular development. Values at each time point were normalized to $100 \%$ at the P1 time-point. Data show mean \pm S.D. of vascular density and Ninj1 expression $(n=4-5)$. (d) Cross-sectioned specimens of the developing eye were stained with an antibody to Ninj1 (green) (P1-P14). Nuclei were counterstained using PI (propidium iodide, red). V: vitreous; R: retina. The white arrowheads indicate a Ninj1-positive cell. (e) Cross-sectioned specimens (P5) were stained with a Ninj1 antibody (red). Differential interference contrast (DIC) microscopy revealed the structure of the ocular specimens. The asterisks indicate the vessel-like structure. (f) Double immunostaining of Ninj1 (red) and VE-cadherin (green) in P5 whole-mounted retinas. Scale bars are $200 \mu \mathrm{m}$ (a) and $50 \mu \mathrm{m}$ (d-f) 
a
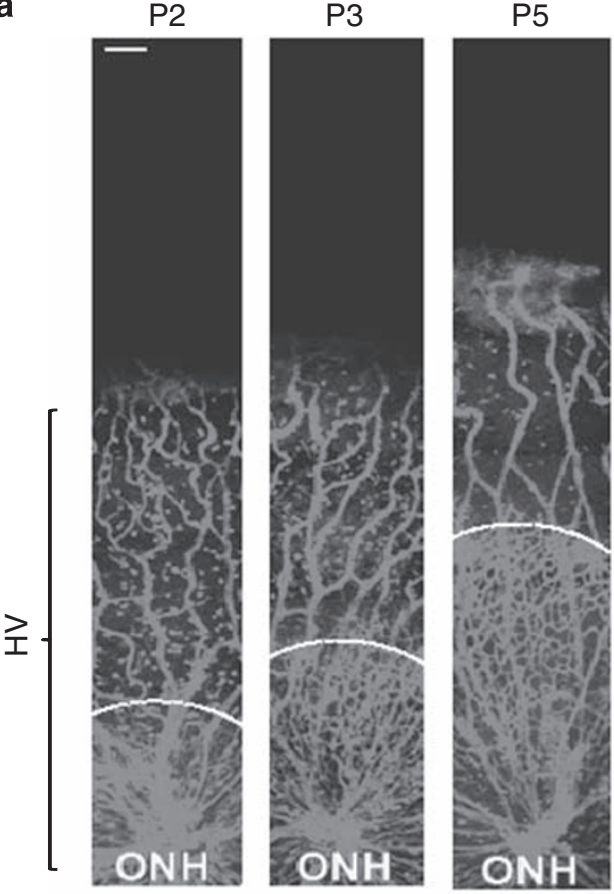

b

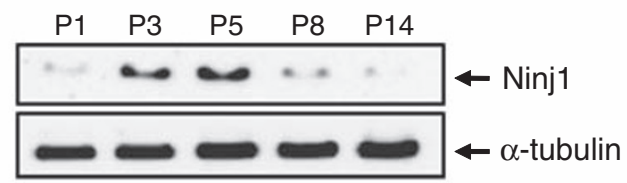

d

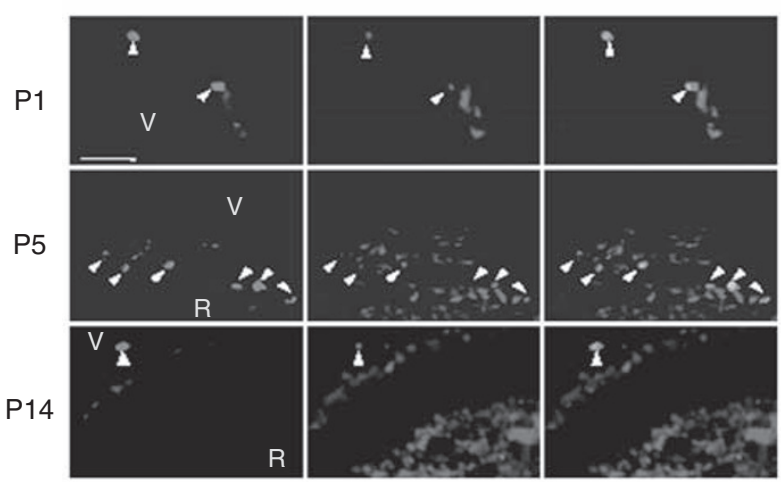

f

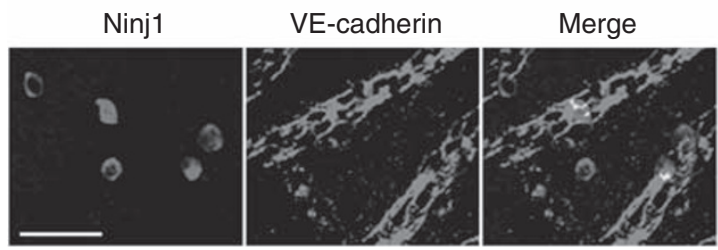

P8
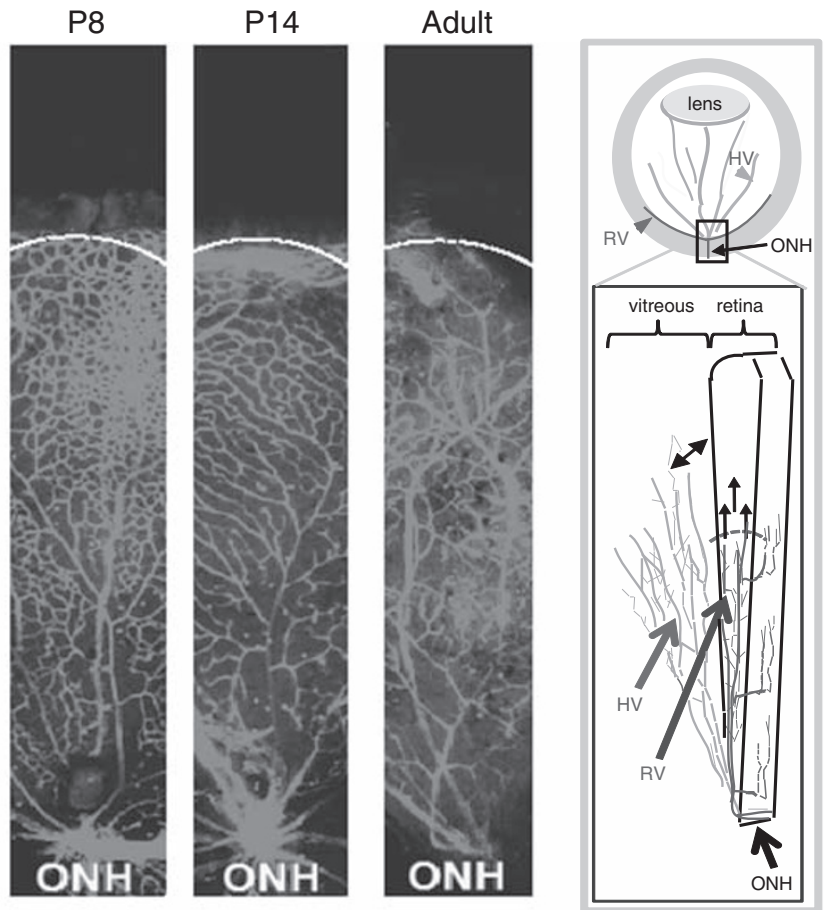

C

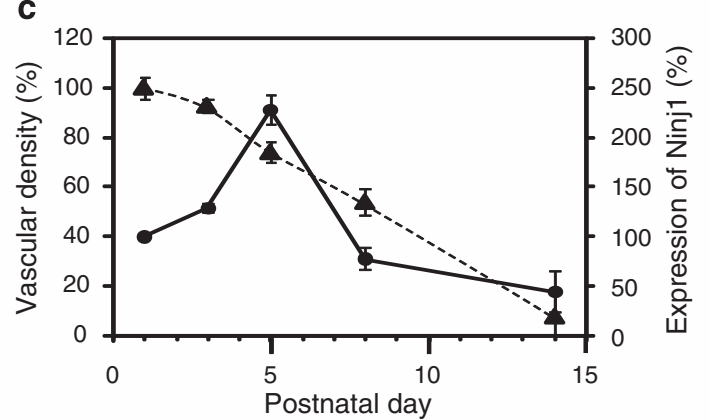

e

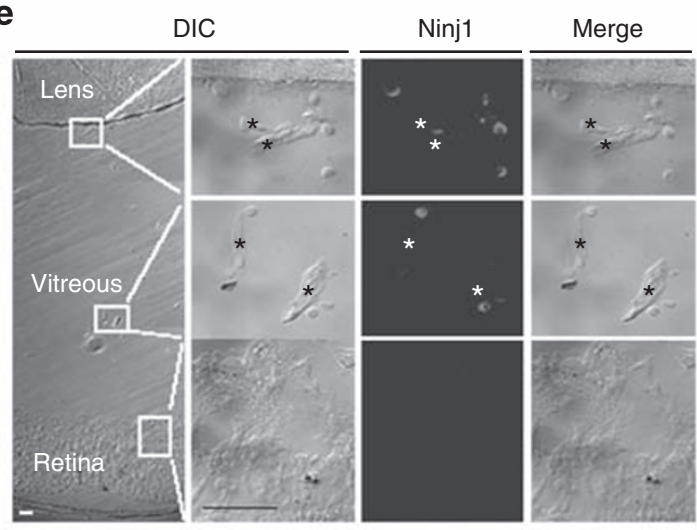


a

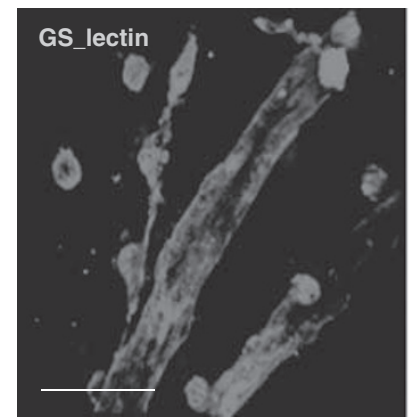

b

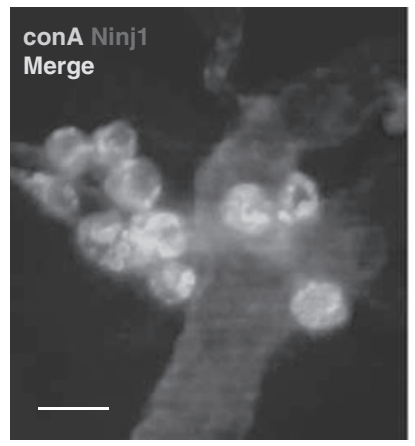

d

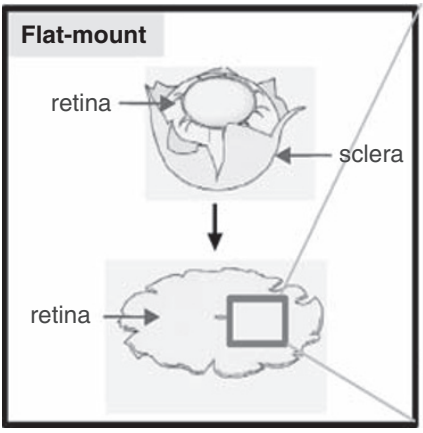

e

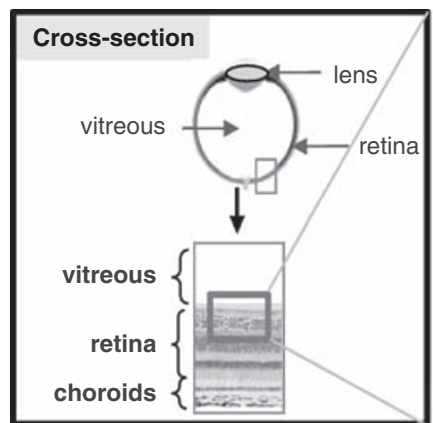

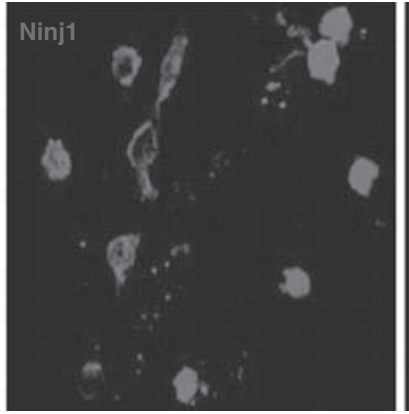
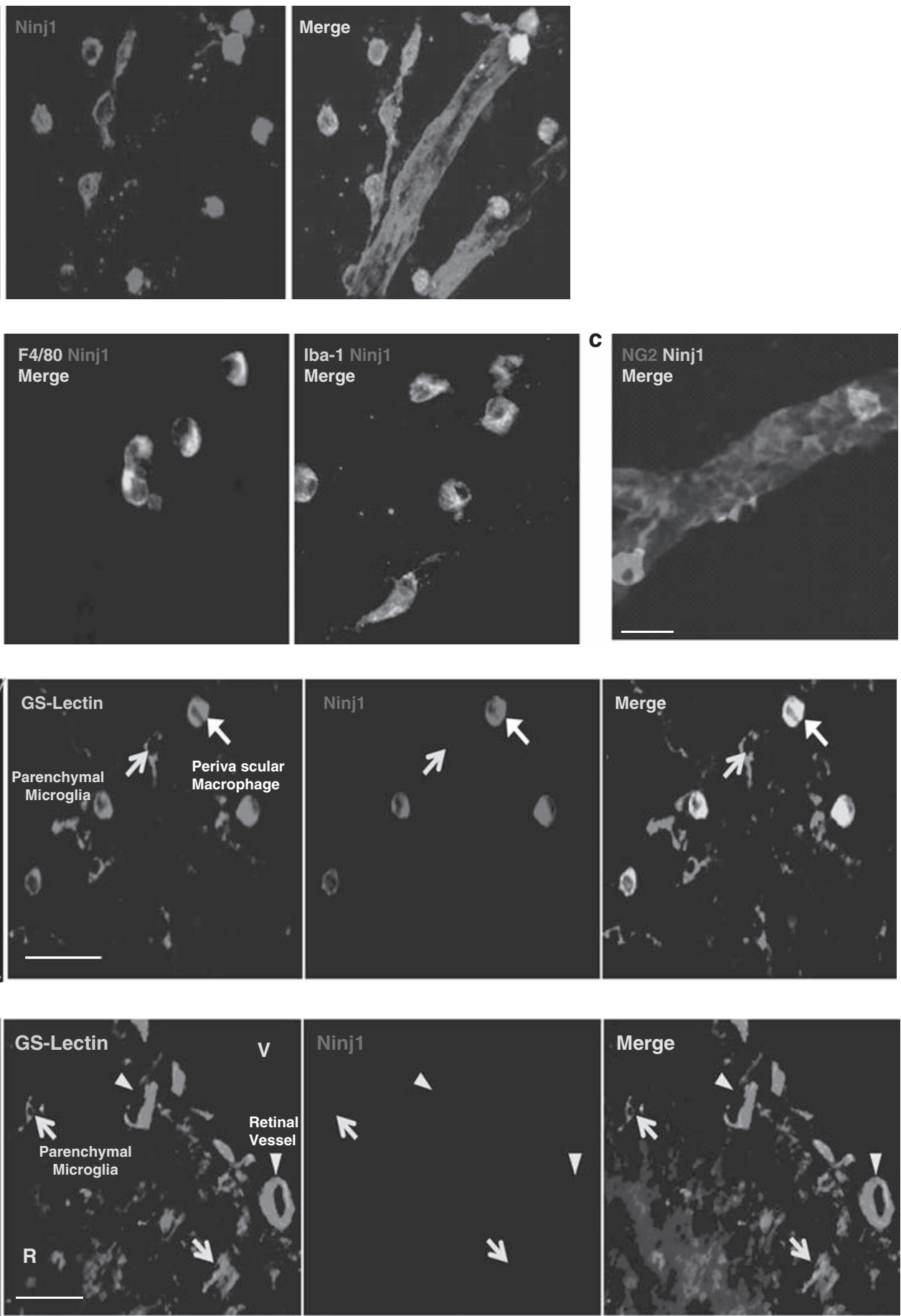

Figure 2 Ninj1 is specifically expressed in perivascular macrophages. (a) Double immunostaining of GS-lectin (green) and Ninj1 (red) in whole-mounted P5 retinas. Overlap of signals is shown in yellow. (b) Double immunostaining of Ninj1 (red) and conA (green) or F4/80 (green); Iba-1 (green) in whole-mounted P5 retinas. Overlap of signals is shown in yellow. (c) Double immunostaining of NG2 (red) and Ninj1 (green) in whole-mounted P5 retinas. (d) Hyaloid vessels were removed using $5 \%$ gelatin ${ }^{7}$ and the remnant whole-mounted P5 eye was stained with GS-lectin (green) and Ninj1 (red). White arrow indicates round-shaped perivascular macrophages and yellow arrow denotes ramified parenchymal microglia. Ninj1 was expressed in perivascular macrophages but not in parenchymal microglia. (e) Immunostaining of cross-sectioned P5 retina with GS-lectin (green) and Ninj1 (red). Ninj1 (red) was not expressed in parenchymal microglia. Nuclei were counterstained using 4'-6-diamidino-2-phenylindole (DAPI, blue). V: vitreous; R: retina. White arrowheads indicate retinal vessels and yellow arrows indicate ramified parenchymal microglia. Scale bars are $50 \mu \mathrm{m}$. The color version of the figure is available online

To identify the type of Ninj1-expressing cells, we performed immunostainings using antibodies specific for macrophages/ endothelial cells (GS-lectin, conA), macrophages (F4/80,
Iba-1), and pericytes (NG2). Ninj1-expressing round cells were positive for GS-lectin (Figure 2a), conA, F4/80, and Iba-1 (Figure 2b), but not for NG2 (Figure 2c), suggesting 
that Ninj1 expression is specific for hyaloid macrophages (hyalocytes) but not for pericytes. Moreover, immunostaining of flat-mounted specimens revealed that Ninj1 was expressed only in round-shaped perivascular macrophages, but not in parenchymal ramified microglia of the retina (Figure $2 \mathrm{~d}$ ). In cross-sections of the retina, GS-lectin stained both retinal vessels and parenchymal microglia in the retinal layer, but these resting microglia did not express Ninj1 (Figure 2e). These results suggest that Ninj1 is specifically expressed in perivascular macrophages around the VECs in the vitreous during HVS regression.

Systemic blockade of Ninj1 during HVS regression and Ninj1 expression in macrophages under endotoxin treatment. To investigate the role of Ninj1 in the macrophage-induced regression of HVS, we performed a double-staining experiment using a Ninj1 antibody and the terminal transferase-mediated dUTP nick-end (TUNEL) labeling. Ninj1 expression coincided spatiotemporally with macrophages neighboring TUNEL-stained VECs (Figure 3a), which suggests that Ninj1 expression may be involved in the regression of HVS. To confirm this possibility, we treated mice with a Ninj1 neutralizing antibody or an isotypic control (IgG) antibody by intraperitoneal (IP) injection. Ninj1 blockage $(1 \mathrm{mg} / \mathrm{kg})$ led to a delay in the regression process when compared with the IgG-injected groups. The number of branching points in Ninj1 antibody-injected groups remained higher than those in the IgG-injected groups at P6 (Figure 3b). Furthermore, the vascular density of Ninj1 antibody-injected animals was higher than that of IgGinjected mice at P6 and this difference was greatly increased at P11 (Figure $3 \mathrm{c}$ ). This indicates that regression of HVS was delayed by neutralizing with Ninj1 antibody compared with control antibody. These findings suggest that Ninj1 may mediate the macrophage-induced regression of HVS during ocular development. To investigate the role of Ninj1 in the activation of macrophages, lipopolysaccharide (LPS) was treated in adult rats and the BV2 macrophage cell line. LPS provoked a remarkable increase in the number of Ninj1-expressing macrophages, but lba-1-positive microglia did not express Ninj1 in the flat-mounted eye (Figure 4a). In cross-sectioned specimens, Ninj1-expressing macrophages appeared in the vitreous and at the border of retina when treated with LPS (Figure 4b). Moreover, LPS treatment increased the expression of Ninj 1 in both adult rat eye (Figure 4c) and BV2 cells (Figure 4d) in a dose-dependent manner. Although many lba-1-expressing macrophages were present both at embryonic stages (E17) and P0, Ninj1 expression was greatly increased in the cells of P0, which starts HVS regression (Supplementary Figure S2). These results suggest that Ninj1 may be important for the migration and activation of macrophages during developmental HVS regression.

The adhesion activity of Ninj1-expressing macrophages. HVS are encapsulated by the extracellular matrix (ECM), which includes type I/IV collagen (col. I/IV) and fibronectin (FN), and we found that Ninj1-expressing macrophages adhere mainly to these ECM components (Figure 5a). In cell-matrix adhesion assays, Ninj1 increased the adhesion of macrophages to ECM, especially in FN and col. IV (Figure 5b). Furthermore, macrophages can directly contact with the plasma membrane of VECs in $\mathrm{HVS}^{26}$ or interact directly with VECs that are undergoing apoptosis. ${ }^{27}$ The close interaction between macrophages and VECs is crucial for signal transduction, such as the Wnt7b pathway. ${ }^{7}$ In cell-cell adhesion assay, Ninj1-transfected macrophages adhered to human umbilical vein endothelial cells 2.2 times more than mock (Mo)-transfected macrophages, which was reversed by Ninj1 antibody treatment (Figure 5c). Many adhesion molecules interact heterophilically as well as homophilically with their family members ${ }^{28-30}$ and the possibility of heterophilic adhesion of Ninj1 has been also suggested. ${ }^{19}$ They showed that peptides containing the adhesion motif of Ninj1 block the basal adhesion of wild-type Jurkat cells, indicating that other molecules with the Ninjurin-like adhesion motif may be involved in heterophilic interaction. ${ }^{19}$ Thus Ninj1-expressing macrophages may adhere to matrix and VECs through heterophilic interactions. Furthermore, Ninj1expressing macrophages often appeared in clusters near the regressing HVS (Figure 5d). Indeed, Ninj1-stably transfected BV2 cells grew as colonies, whereas wild-type BV2 cells did not (Figure 5e). Moreover, these aggregations were enhanced in Ninj1-transfected BV2 cells compared with other conditions (Figure 5f), suggesting that Ninj1 enhances the homophilic adhesion in macrophages. Such cell clusters may promote more effective signal transduction to their target cells. However, as Ninj1 enhances the adhesion to ECMs and VECs as well as other macrophages, Ninj1-expressing cells exist in various forms, such as one cell (Figures 1d-f, 2a and $5 \mathrm{a}$ ), two cells (Figure $2 \mathrm{~b}$, middle and right), or aggregates (Figure $2 b$, left and) during HVS regression. Accordingly, these data demonstrate that Ninj1 promotes the cellmatrix and cell-cell adhesion of macrophages and may result in synergistic concentration of death signaling through the narrowing of the gap between macrophages and VECs in HVS.

Ninj1 activates the Wnt-Ang signaling, resulting in apoptosis of VECs. It has previously been demonstrated that the Wnt-Ang signaling pathway is important in the programmed cell death of HVS. ${ }^{6,7}$ Macrophages secrete a Wnt7b ligand which is required for cell-cycle entry and apoptosis of HVS. $^{7}$ In addition, pericytes produce Ang2, which inhibits the PI3-kinase-Akt survival signaling pathway in VECs and promotes Wnt7b signaling in macrophages; this in turn leads to cell death and regression of vessels. ${ }^{6,7}$ These facts led us to investigate the relationship between Ninj1 and the Wnt-Ang pathway. To examine such relationship, we transfected BV2 cells with either mock (Mo) or Ninj1 DNA. Wnt7b expression was increased in the Ninj1-transfected macrophages compared with Mo-transfected groups (Figure 6a). Ninj1 overexpression increased Wnt7b expression in a single macrophage, as well as in aggregated macrophages, suggesting that cell-cell adhesion of macrophages is not required for Ninj1-induced expression of Wnt7b (Figure 6b). Furthermore, silencing of Ninj1 strongly inhibited the expression of Wnt7b (Figure 6c). These results collectively show that Ninj1 expression is capable of inducing the expression of Wnt7b. To investigate the molecular mechanism 


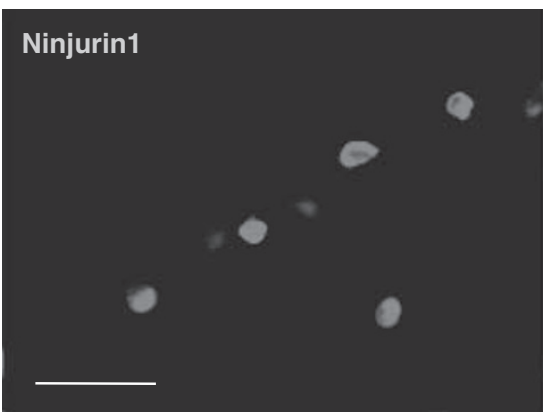

b

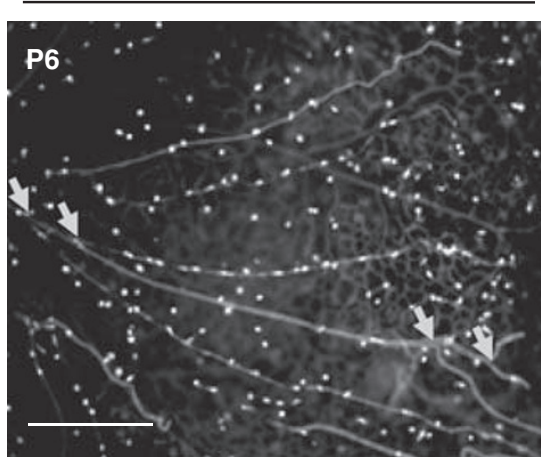

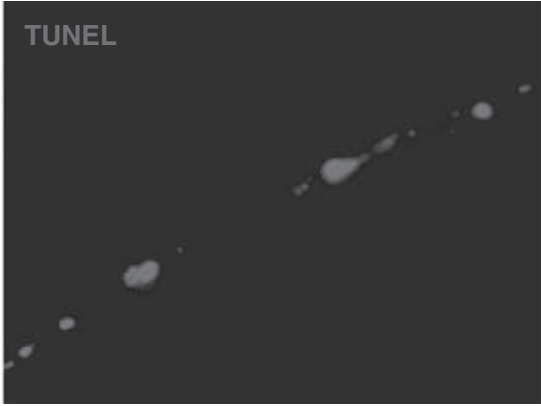

Ninj1 Ab

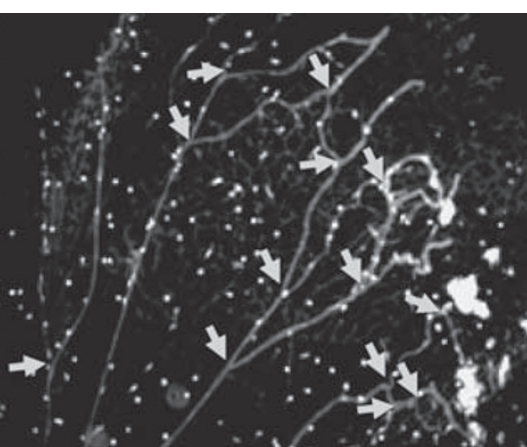

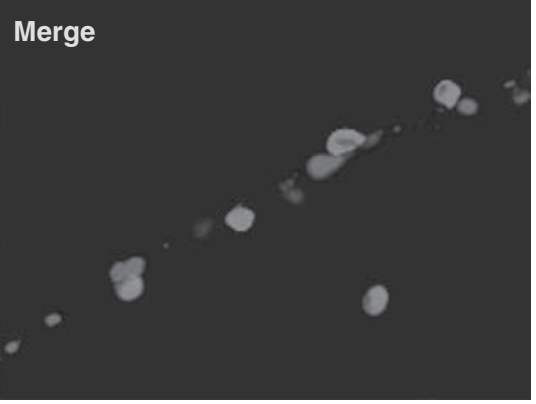

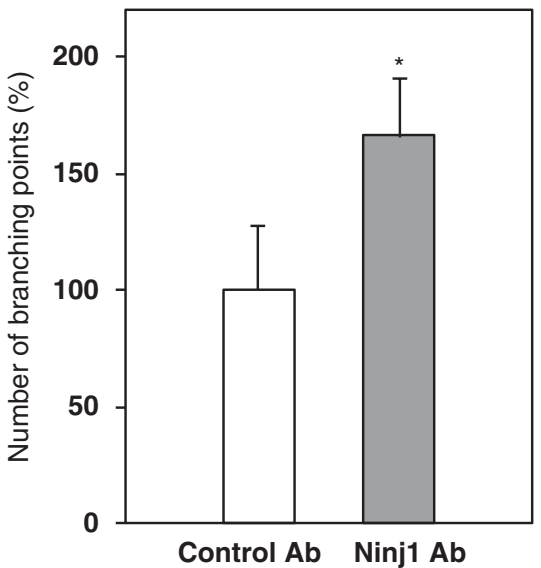

C
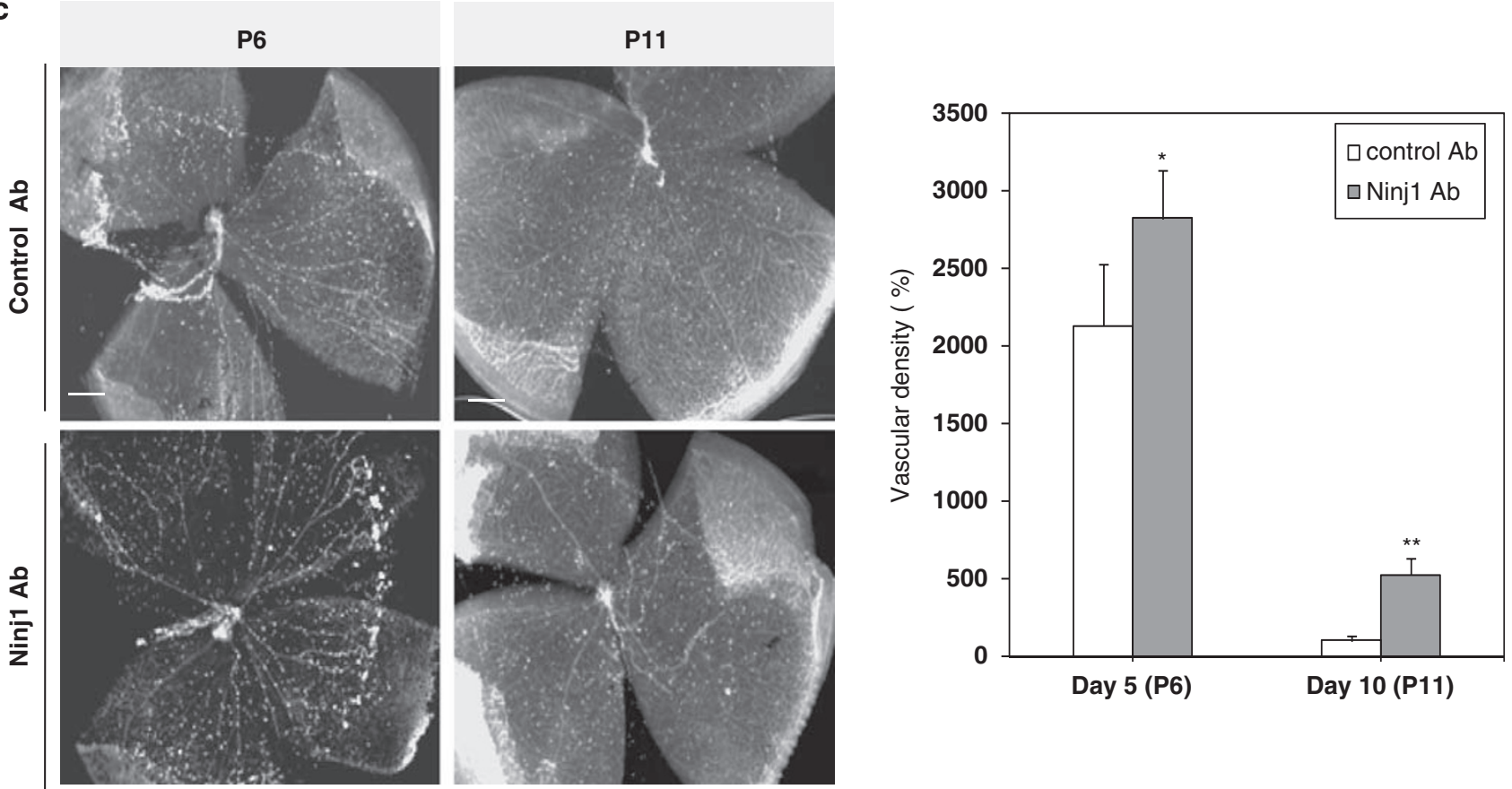

Figure 3 Ninj1 is important for macrophages-induced regression. (a) Ninj1-expressing macrophages adhered to TUNEL-positive endothelial cells of P8 eye. (b) GS-lectin staining of P6 whole-mounted mouse retinas treated with control antibody or Ninj1 neutralizing antibody. Yellow arrows indicate the branching point (left). Quantitative analysis of branching points at P6 revealed a significant difference in the number of vascular branching points between the groups treated with Ninj1 antibody (Ninj1 Ab) and the control antibody (Control Ab; right). Value was normalized to $100 \%$ at density of control (isotype injected) mice on P6. Error bar represents S.D.; ${ }^{*} P<0.05$. (c) GS-lectin staining of P6 and P11 whole-mounted retinas. There was a significant difference in vascular density between the group treated with Ninj1 Ab and Control Ab (left). Vascular density of ICR mice was measured after neutralizing with antibodies (right). Values at each time point were normalized to $100 \%$ at density of control (isotype injected) mice on $\mathrm{P} 11$. ${ }^{*} P<0.05$; ${ }^{* \star} P<0.01$ (unpaired $t$-test). Data (mean \pm S.E.M.) are representative of one experiment with 8-10 mice per group. Scale bars are $50 \mu \mathrm{m}(\mathbf{a}), 1 \mathrm{~mm}(\mathbf{b}$ and $\mathbf{c}$ ) 
a
PBS

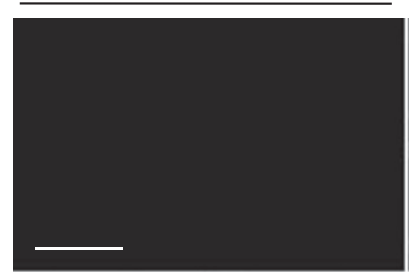

1.

Iba-1

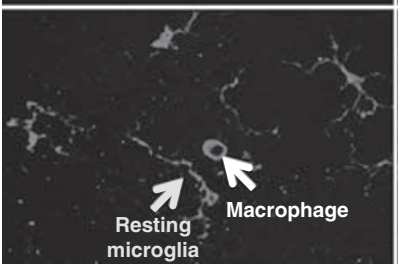

b

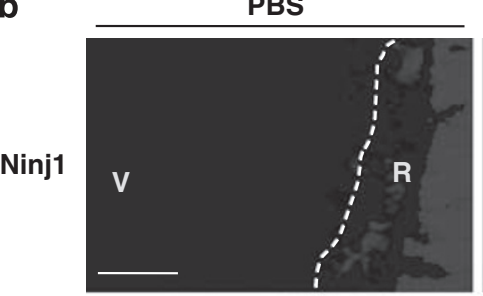

PBS

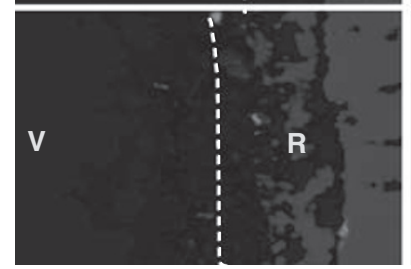

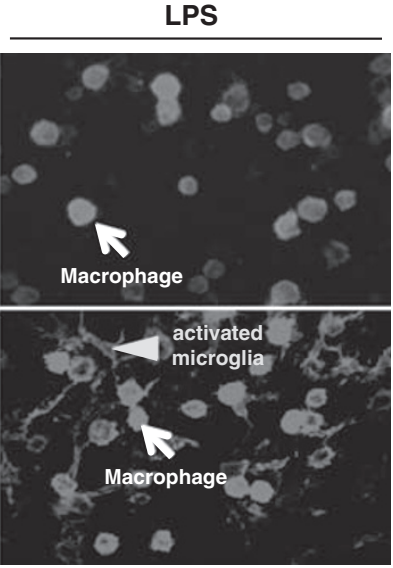

LPS

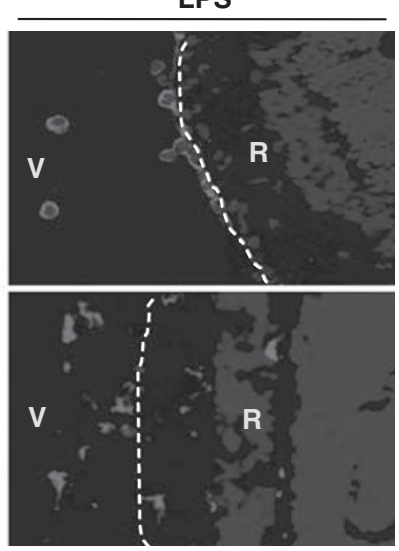

c

Eye

LPS :

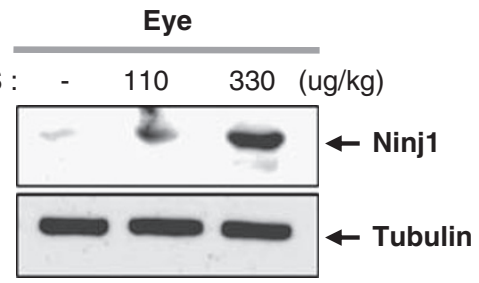

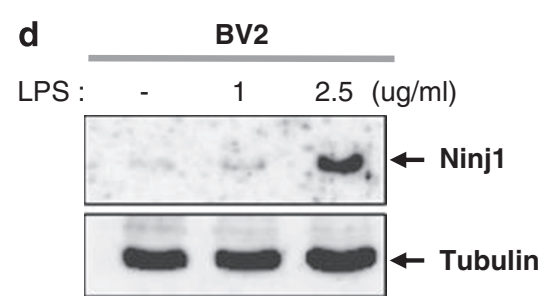

Figure 4 Ninj1 expression in macrophages under LPS treatment in vivo and in vitro. (a) Immunostaining of adult whole-mounted retinas with antibodies for Ninj1 (green) and Iba-1 (green). Ninj1-positive cells (green, upper panel) appeared only after IP injection of lipopolysaccharide (LPS) in rat adult retina (7 weeks). Iba-1 immunostaining of flat-mounted specimens (green, lower panel) showed that LPS promotes the influx of ovoid macrophages and the transition from ramified microglia to activated microglia. Yellow arrow: the resting parenchymal microglia; yellow arrowhead: activated parenchymal microglia; white arrow: macrophages. (b) Immunostaining of adult cross-sectioned retinas with antibodies for Ninj1 (red) and Iba-1 (red). Ninj1-positive rounded macrophages (red, upper panel) were observed in vitreous and at the retinal border only after LPS injection. Iba-1 staining in cross-sectioned specimens (red, lower panel) showed entry and/or activation of macrophages and microglia by LPS treatment. Nuclei were counterstained using DAPI (blue). V: vitreous; R: retina. White dotted lines indicate retinal boundary. (c) Ninj1 expression in eye lysates after 0-330 $\mu \mathrm{g} / \mathrm{kg}$ of LPS injection in adult rat (7 weeks). (d) Ninj1 expression in BV2 cells after $0-2.5 \mu \mathrm{g} / \mathrm{ml}$ of LPS treatment. Scale bars are $50 \mu \mathrm{m}$ (a and $\mathbf{b})$. The color version of the figure is available online

underlying this phenomenon, we examined whether Ninj1 affected the activation of p38, p44/42 MAPK and JNK. Ninj1 markedly increased the phosphorylation of p38 but had no effect on that of p44/42 and JNK (Figure 6d). Furthermore, a specific inhibitor of p38 MAPK (SB203580) led to a decrease in Ninj1-induced Wnt7b expression (Figure 6e). To establish more conclusively the link between Ninj1, p38 MAPK, and Wnt7b, we treated cells with siRNA of Ninj1, which resulted in the downregulation of the phosphorylation of p38 MAPK as well as the expression of Wnt7b (Figure 6f). These results suggest that Ninj1 increases the Wnt7b expression via p38 MAPK pathway. To further elucidate the mechanism, we examined the expression of several transcription factors containing the DNA binding sites in the Wnt7b promoter such as TTF-1, p53, and GATA-6. Although TTF-1, p53, and GATA-6 have been reported to increase the transcription of Wnt7b, we could not detect any changes in their expression levels in Ninj1-overexpressing macrophages (Supplementary Figure S3), suggesting that unknown transcription factor(s) may be involved in the Wnt7b expression mediated by Ninj1. Altogether, these data indicate that Ninj1-expressing macrophages might increase Wnt7b production through p38 MAPK 

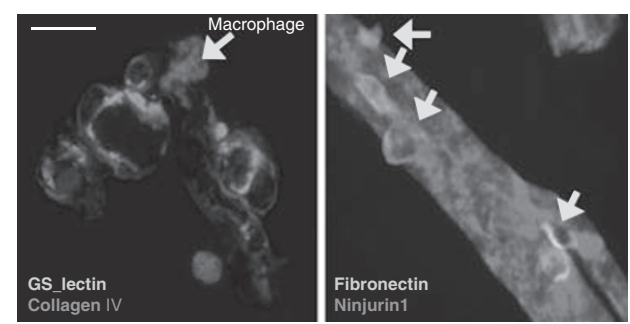

b

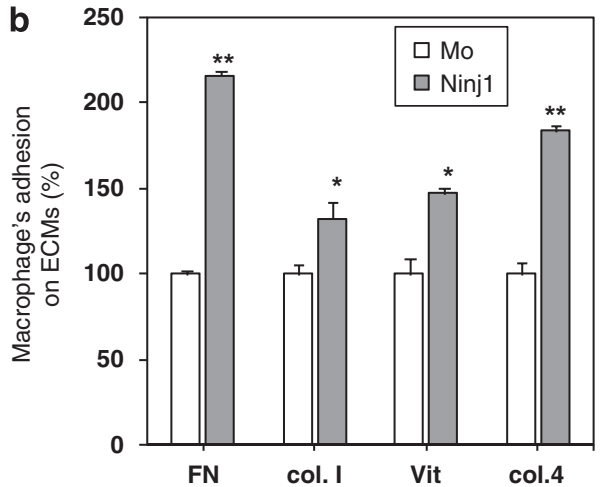

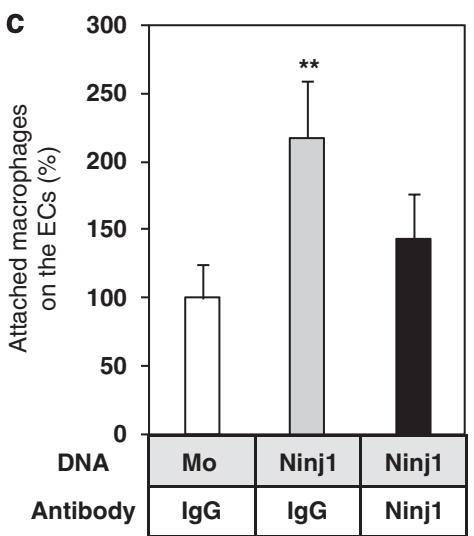

e

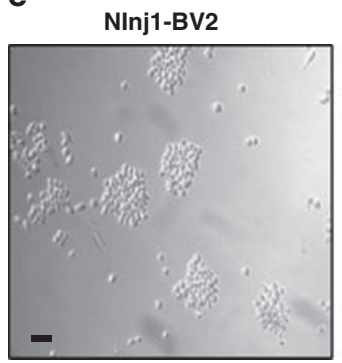

BV2

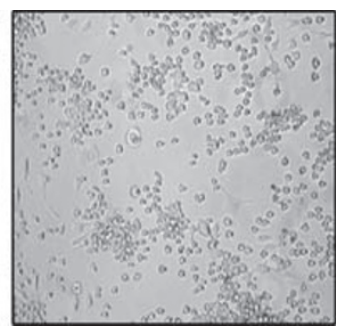

d

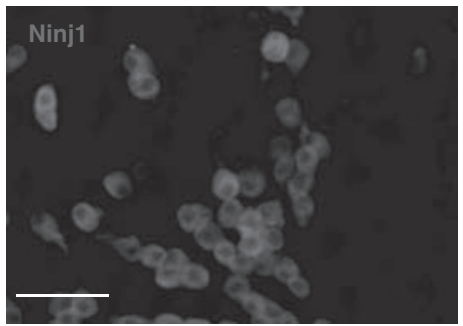

f

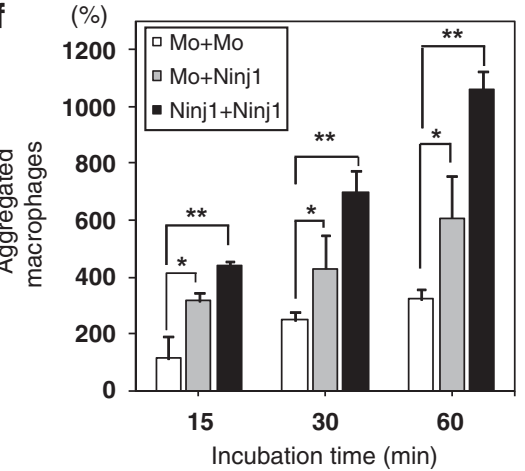

Figure 5 Ninj1 increases cell-cell and cell-matrix adhesion. (a) Double staining of GS-lectin (green) and type IV collagen (red), or fibronectin (green) and Ninj1 (red). Yellow arrows show macrophages attached to VECs in HVS. (b) Cell-to-matrix adhesion assay using BV2 cells expressing either mock (Mo) or Ninj1 DNA. FN: Fibronectin; col.I: type I collagen; Vit: vitronectin; col.IV: type IV collagen. Cell adhesion values are expressed as relative to the adhesion of Mo-transfected cells, normalized to $100 \%$. Error bar represents S.D.; ${ }^{*} P<0.05,{ }^{\star} * P<0.01$. (c) After transient transfection with Mo or Ninj1 DNA, BV2 cells were neutralized with Ninj1 antibody or control IgG. Adhesion of macrophages to endothelial cells was analyzed quantitatively. Adhesion degree is expressed as relative to Mo-transfected cells that were treated with control lgG antibody, normalized to $100 \%$. Values are mean \pm S.D. for each group $(n=10-12) ;{ }^{*} P<0.01$. (d) Staining of Ninj1 (red) at P3. Ninj1-expressing macrophages often formed aggregates around HVS. (e) BV2 cells stably transfected with Ninj1 (Ninj1-BV2) and wild-type BV2 cells (BV2) were cultured for 2 days. Ninj1-BV2 grew to form large aggregates. (f) Quantitative analysis of the aggregation using mixed cell populations. Mo: Mock-transfected BV2 cells; Ninj1: Ninjurin1-transfected BV2 cells. Values were calculated as relative to the aggregating degree when Mo-transfected cells were mixed with other Mo-transfected cells (Mo + Mo condition) after 15 min incubation and normalized to $100 \%$. Error bar represents S.D.; ${ }^{*} P<0.05,{ }^{* \star} P<0.01$. Scale bars are $25 \mu \mathrm{m}(\mathbf{a})$ and $50 \mu \mathrm{m}(\mathbf{d}$ and $\mathbf{e})$. The color version of the figure is available online

phosphorylation. Wnt7b secreted from macrophages can activate Wnt7b pathway of the adjacent VECs, resulting in enhancing the sensitivity to cell death. ${ }^{7}$

Next, we investigated whether Ninj1 influenced Ang pathway in pericytes. As it has been reported that Wnt7b can be secreted out of cells and exist in conditioned medium (CM) in quantities detectable in vitro, ${ }^{31}$ we used the $\mathrm{CM}$ in the in vitro assay. The CM from Ninj1-transfected macrophages (Ninj1-
$\mathrm{CM}$ ) decreased Ang1 and increased Ang2 in pericytes when compared with the CM from Mo-transfected macrophages (Mo-CM; Figure 7a). Although the mechanism which Ninj1 regulates differentially the expression of Ang1 and Ang2 in pericytes is currently unclear, these results suggest that Ninj1 may induce apoptosis of HVS through differential regulation of the Ang pathway in pericytes. Collectively, Ninj1 can enhance Wnt7b in macrophages and also upregulate Ang2 and 
a

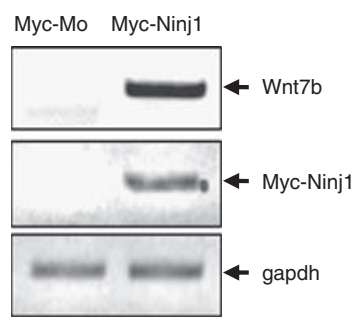

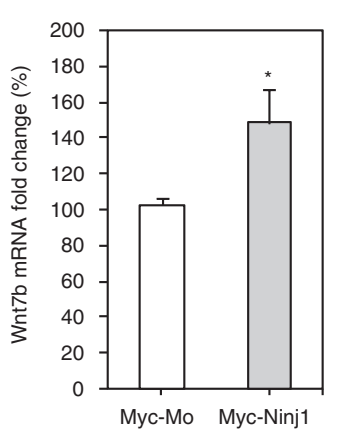

b Myc

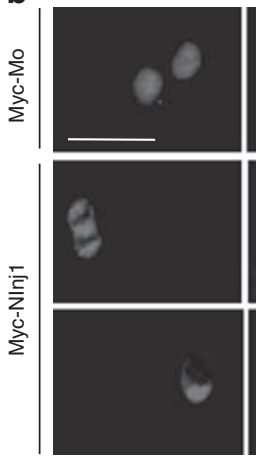

$\sqrt{6}$
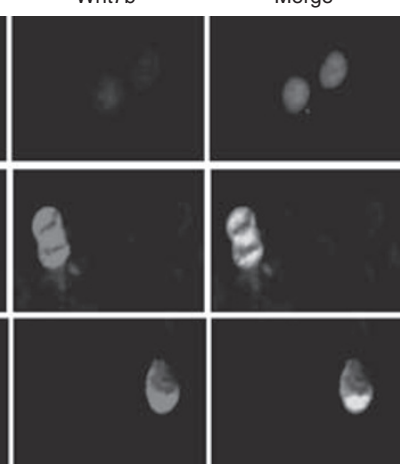

C

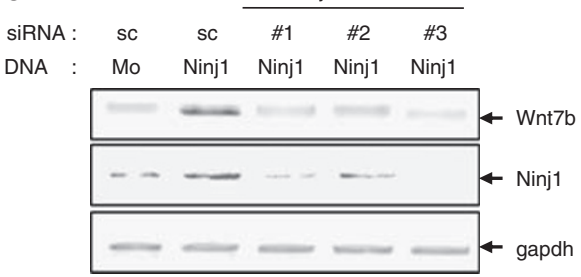

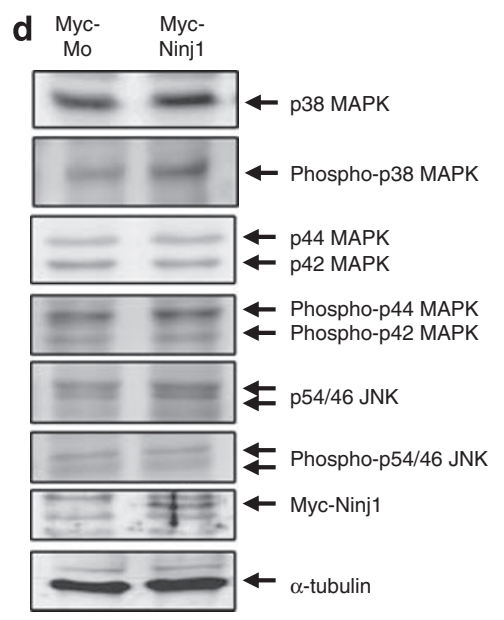
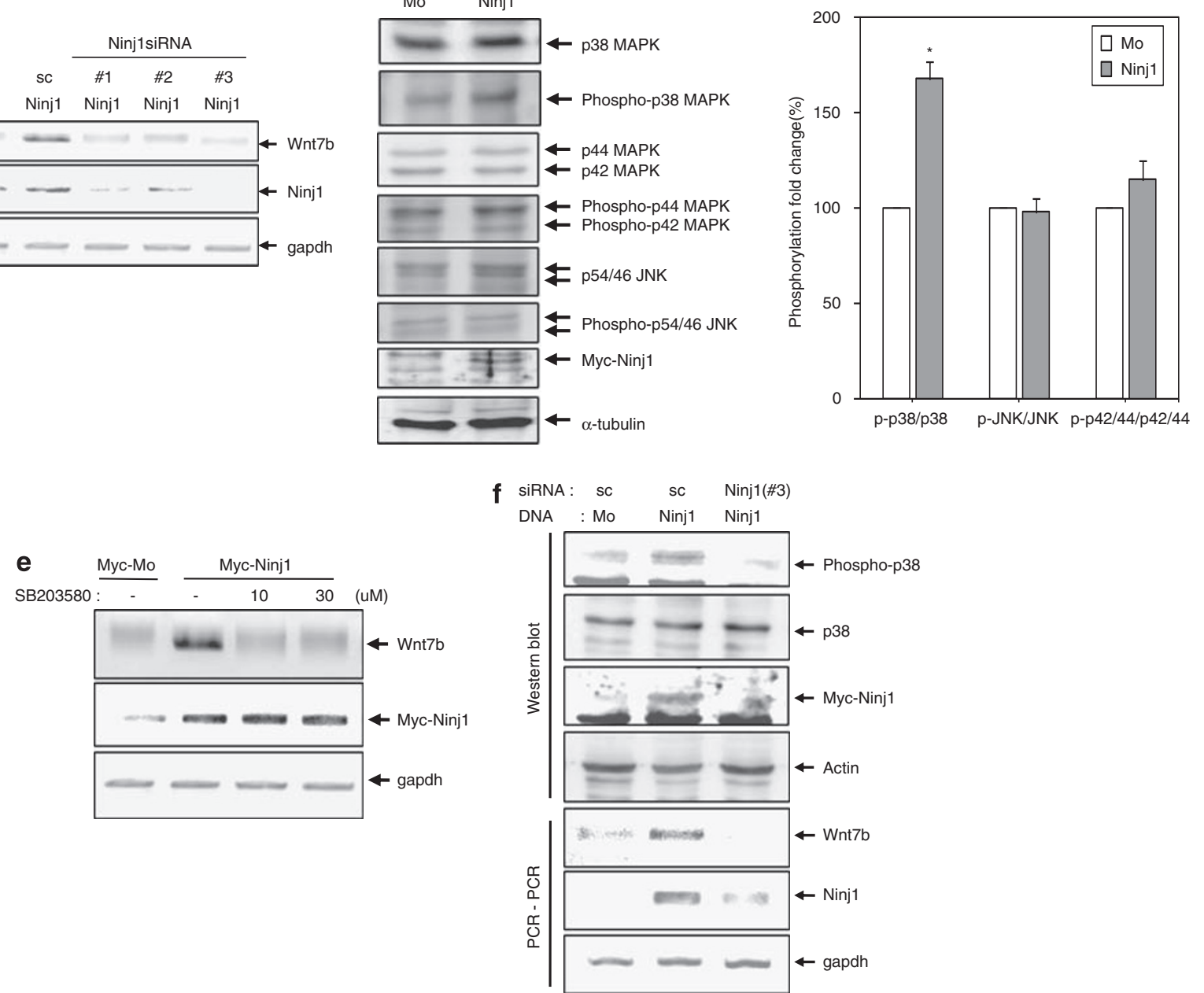

Figure 6 Ninj1 enhances the Wnt7b expression in macrophages. (a) RT-PCR of Wnt7b in BV2 cells expressing either myc-tagged mock (Myc-Mo) or myc-tagged Ninj1 DNA (Myc-Ninj1; left). Gapdh was used as an internal control. Real-time PCR of Wnt7b in BV2 cells expressing either Myc-Mo or Myc-Ninj1 (right). Error bar represents S.D.; ${ }^{*} P<0.05$. (b) Immunostaining of c-Myc (red) and Wnt7b (green) in BV2 cells expressing either Myc-Mo or Myc-Ninj1. After transient transfection, BV2 cells were cultured for 2 days in DMEM containing 1\% serum. (c) RT-PCR in BV2 cells expressing Myc-Mo or Myc-Ninj1 followed by siRNA treatment. Depletion of Ninj1 by three different short interfering RNAs (siRNAs) downregulated the expression of Wnt7b in BV2 cells. (d) Effect of Ninj1 on the phosphorylation of p38, p44/p42, and JNK. Western blot analysis was performed using total cell lysates from Myc-Mo or Myc-Ninj1 expressing BV2 cells (left). Quantification of phosphorylation status of p38, p44/p42, and JNK. Values were normalized to $100 \%$ at the phosphorylation of kinases in BV2 cells expressing Myc-Mo. Error bar represents S.D.; ${ }^{*} P<0.05$ (right). (e) Ninj1-induced Wnt7b expression was downregulated in a dose-dependent manner by a p38 inhibitor, SB203580. RT-PCR was performed using BV2 cells. (f) Western blot and RT-PCR in BV2 cells expressing Myc-Mo or Myc-Ninj1 followed by siRNA treatment. Scale bar is $25 \mu \mathrm{m}$ (b)

downregulate Ang1 in pericytes, which induces apoptosis of VECs. To examine the effect of the Ang pathway on macrophages, macrophages were serum starved overnight
(16 h) to annul the influence of other factors, and were then incubated for $24 \mathrm{~h}$ with recombinant human Ang1 (rh-Ang1) and Ang2 (rh-Ang2) proteins, or with serum-free medium as a 
a
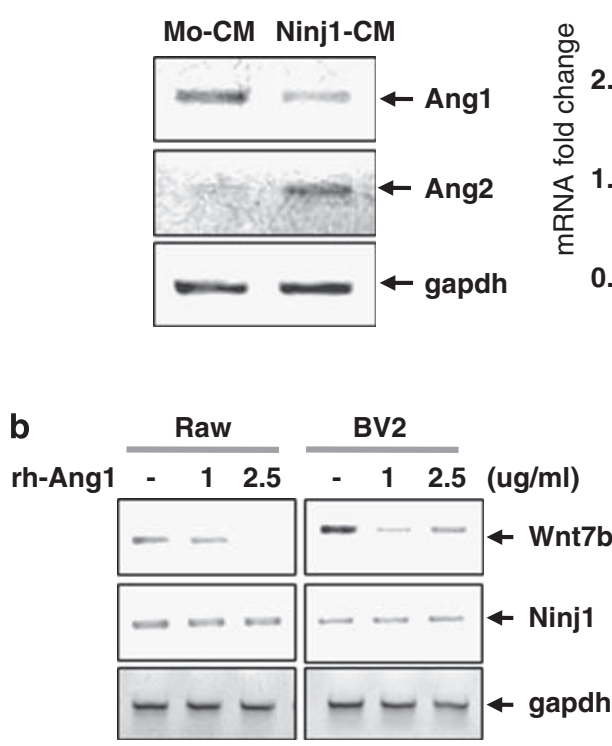
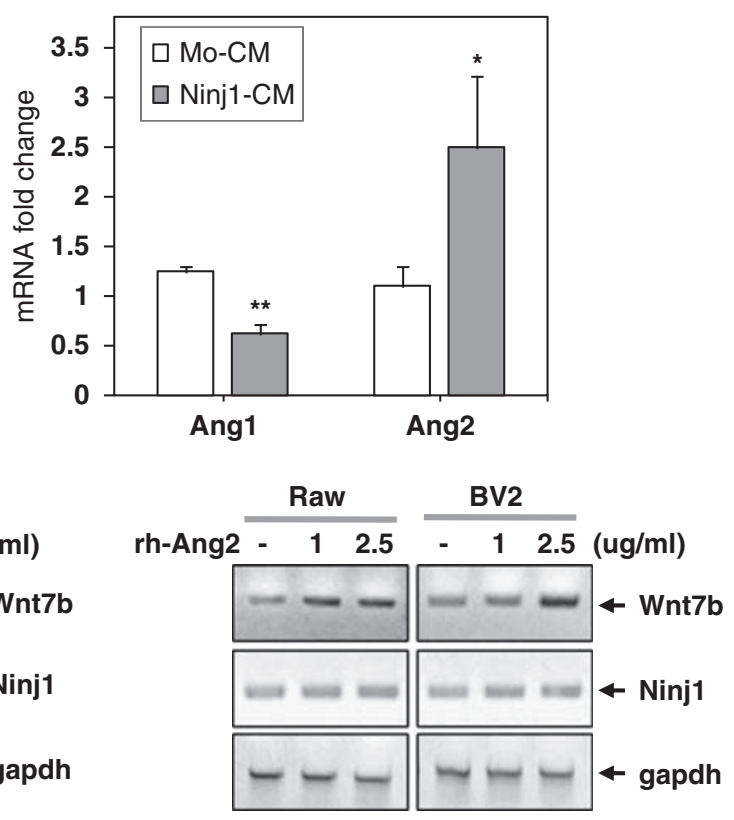

+Ang2
C

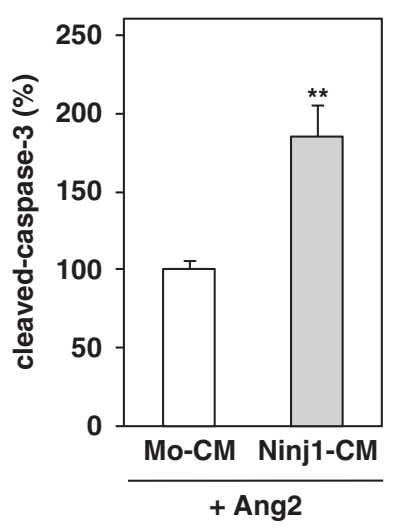

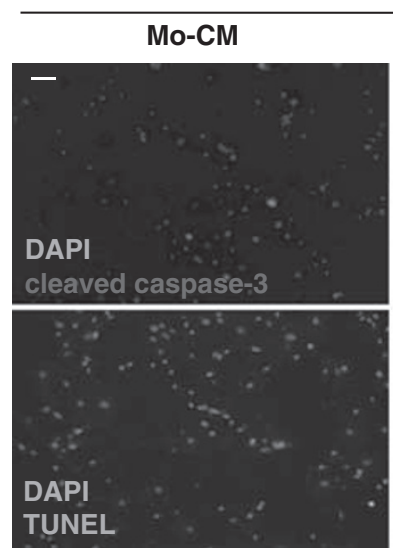

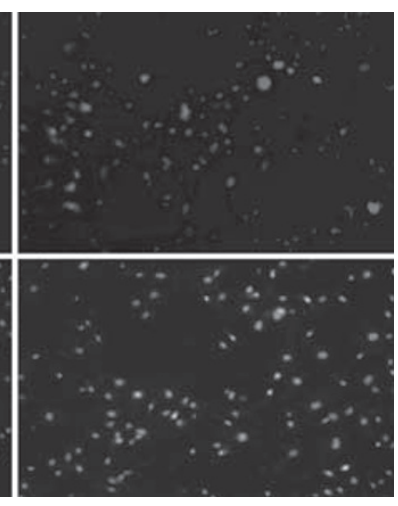

Figure 7 Ninj1 regulates the Ang pathway and enhanced apoptosis of endothelial cells in the presence of Ang2. (a) RT-PCR of Ang1 and Ang2 was performed in human pericytes treated for $24 \mathrm{~h}$ with conditioned media of mock-transfected (Mo-CM) or Ninj1-overexpressing BV2 cells (Ninj1-CM; left). Gapdh was used as an internal control. Real-time PCR of Ang1 and Ang2 was performed in human pericytes treated with Mo-CM or Ninj1-CM (right). Values are mean \pm S.D. for each group ( $n=3$ ); ${ }^{*} P<0.05$, ${ }^{*} P<0.01$. (b) RT-PCR of Wnt7b and Ninj1 in Raw264.7 and BV2 cells, in the presence or absence of recombinant human Ang1 (rh-Ang1; upper). RT-PCR of Wnt7b and Ninj1 in Raw264.7 and BV2 cells, in the presence or absence of recombinant human Ang2 (rh-Ang2; lower). (c) Western blotting of cleaved-caspase3 in HUVECs treated for $24 \mathrm{~h}$ with Mo-CM or Ninj1-CM in the presence of rh-Ang2 (left). Error bar represents S.D.; ${ }^{\star \star} P<0.01$. Immunostaining of the cleaved-caspase3 (red) and TUNEL (green) in HUVECs treated for $24 \mathrm{~h}$ with Mo-CM or Ninj1-CM in the presence of rh-Ang2 (right). Nuclei (blue) were counterstained using DAPI. Scale bar is $50 \mu \mathrm{m}$ (c). The color version of the figure is available online

control. Although there were no changes in the expression of Ninj1 under these conditions, Wnt7b was downregulated by rhAng1 and was upregulated by rh-Ang2 in a dose-dependent manner (Figure 7b). These results were consistent with a previous report stating that the injection of Ang2 intravitreally in vivo upregulates $W n t 7 b .{ }^{6}$ Therefore, these data indicate that Ang1 and Ang2 do not influence the expression of Ninj1, but can regulate Wnt7b expression differentially. Finally, we confirmed the apoptotic-inducing activity of Ninj1 in the presence of Ang2. We treated Mo-CM or Ninj1-CM into VECs with rh-Ang2 to mimic the in vivo condition in which macrophages, pericytes, and VECs interact. Furthermore, Ang2 not only stimulates Wnt ligand production in macrophages but also inhibits survival signaling in VECs during HVS regression. ${ }^{6}$ Ninj1-CM increased cleaved caspase-3 activity of VECs compared with Mo-CM in the presence of rh-Ang2 (Figure 7c, left). In addition, Ninj1-CM increased both the cleaved caspase-3- and TUNEL-stained VECs under cotreatment with Ang2 (Figure 7c, right). These data suggest that Ninj1 enhances apoptosis of VECs in the presence of Ang2 and may increase Ang2-induced programmed cell death of VECs during early ocular development.

\section{Discussion}

Ninjurins, Ninj1 and Ninj2, are small adhesion molecules and are upregulated in response to nerve injury. ${ }^{18}$ However, they 
do not contain comparable adhesive motifs nor do they interact with each other. ${ }^{20}$ Ninj1 is broadly expressed not only in injured neurons but also in epithelial cells, indicating that Ninj1 may be important in the development and function of other tissues. ${ }^{19}$ Recently, it has been reported that Drosophila $\mathrm{NijA}$ is expressed in tracheal cells and regulates the tracheal remodeling by interaction with MMP1. ${ }^{24}$ The Drosophila tracheae are epithelial tubes and the underlying molecular mechanisms of the Drosophila tracheal (respiratory) patterning and differentiation are similar to that of blood vessel (and lung) development in vertebrates. ${ }^{32}$ Furthermore, the regulation of extracellular matrix (ECM) and cell-cell interaction is important for proper vascular remodeling. ${ }^{33}$ Therefore, these reports suggest that, an adhesion molecule, Ninj1 may be involved in cell-cell interaction and have an important role in the process of vascular remodeling in higher vertebrates. In this study, we demonstrate that Ninj1-expressing macrophages induce programmed cell death of VECs by cooperation between Wnt and angiopoietin pathway. Interestingly, we found that Ninj 1 is expressed only in perivascular macrophages whose expression appeared to be concerned with vascular density regression curve. Indeed, systemic blockade with anti-Ninj1 antibody showed delayed regression of HVS suggesting that Ninj1 has a role in HVS regression. During regression period, Ninj1-expressing macrophages appeared in clusters, were common near to the regressing HVS, and often directly contacted with VECs. In in vitro adhesion assays, we demonstrated that Ninj1 might promote cell-tomatrix and cell-to-cell adhesion. These adhesion abilities may offer their target cells a more effective signal transduction by narrowing the gap between macrophages and VECs of HVS and concentrating the signals such as Wnt7b. Moreover, Ninj1 enhanced Wnt7b expression, which makes VECs more sensitive to death stimuli via cell-cycle entry. On the other hand, Ninj1-CM stimulated Ang2 but decreased Ang1 differentially in pericytes, resulting in a switch from survival to death of VECs. These data proposed the role of Ninj1 in macrophage-induced programmed cell death of VECs. We confirmed this possibility by combinational treatment with both $\mathrm{CM}$ and Ang2 in which Ninj1 enhances Ang2-induced programmed cell death of VECs.

It has been generally accepted that macrophages have an essential role for homeostasis, inflammation, and injury. ${ }^{34}$ These activities are induced through the activation process after exposure to extrinsic signals, including cytokines and bacterial cell wall products. In general, transient induction or enhanced expression of a set of genes is believed to contribute to the macrophage activation. In this respect, Ninj1 is similar to these genes in that its expression is transient and enhanced in macrophages during HVS regression or after LPS treatment. When regression signals or injuries occur, Ninj1 may facilitate the transport of macrophages to the site of target, where it participates in cell adhesion and provides more effective signal to target cells. Activated macrophages also produce many molecules including basic fibroblast growth factor (bFGF), transforming growth factor- $\beta$ (TGF- $\beta$ ), and proteases. $^{35}$ Especially, degrading enzymes (e.g., collagenase and tissue-type plasminogen activator) and TGF- $\beta$ can change the composition of the ECM not only during angiogenesis, but also during degeneration or break- down of vascularized organs. ${ }^{36}$ After Ninj1-expressing macrophages adhere to ECM of HVS, these cells might modulate the ECM and then make VECs more accessible, leading to morphogenesis.

It is difficult to distinguish between perivascular macrophages and activated parenchymal microglia and they are often designated as macrophage/microglial cells. ${ }^{37}$ To differentiate microglia from macrophages, immunostaining and flow cytometry have been performed using several surface markers such as CD45, a panleukocyte marker, and CD11b, a protein expressed on granulocytes and macrophages. ${ }^{38}$ CD45 and CD11b are also expressed by microglia; however, CD45 is present at lower levels than in macrophages and granulocytes. However, it is still difficult to clarify the cell types via conventional immunohistochemical techniques. Interestingly, we found that Ninj1 is expressed only in round-shaped perivascular macrophages but not in ramified and activated microglial cells, suggesting that Ninj1 might be a specific antigen for activated perivascular macrophages.

Altogether, our results suggest that Ninj1 plays an important role in the close interaction between macrophages and VECs and also in the activation of apoptotic pathway during the regression of HVS. This is summarized in Figure 8, where it

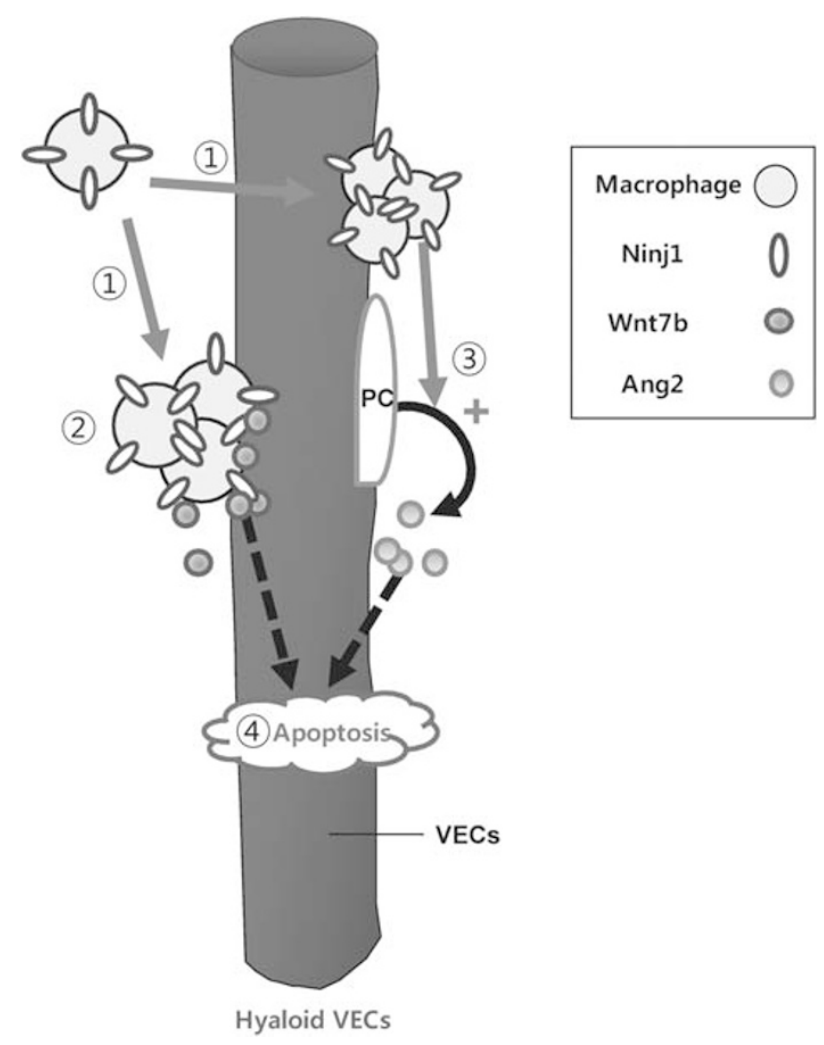

Figure 8 Proposed mechanism of Ninj1-mediated regression of HVS. Ninj1 is expressed in macrophages, thus promoting the cell-cell interaction and formation of clusters (1). Ninj1-expressing macrophages may produce Wnt7b into plasma membrane and partially secrete Wnt7b into vitreous which influences on pericytes (PC) and endothelial cells, especially more effective on adjacent cells (2). Moreover, Ninj1 upregulates the expression of Ang2 in pericytes (3). Upregulation of Wnt7b induces cell-cycle entry and Ang2 reduces cell stability, which lead to apoptosis of the adjacent VECs of HVS (4) 
shows that Ninj1 is transiently expressed in macrophages, thus enhancing adhesion, aggregation, and Wnt7b expression. Moreover, Ninj1 upregulates the expression of Ang2, which induces the destabilization of VECs in HVS. Therefore, upregulation of Wnt7b induces cell-cycle entry and enhanced Ang2 reduces cell stability, which lead to apoptosis of the VECs of HVS. The transient expression of Ninj1 and its apoptosis-inducing function in macrophages suggest that Ninj1 plays an important role in the proper development and function of a number of tissues such as tail or limb regression mediated by macrophages.

\section{Materials and Methods}

Animals. Specific pathogen-free pregnant Sprague-Dawley (SD) rats and ICR mice were purchased from Samtako Bio Korea and maintained in pathogen-free conditions in the animal-housing facilities of the College of Pharmacy, Seoul National University (Seoul, Republic of Korea) for the duration of the experiments. All animal studies were approved by the Committee for Care and Use of Laboratory Animals at the Seoul National University, according to the Guide for Animal Experiments edited by Korean Academy for Medical Sciences (SNU-071019-3 and SNU-090119-3).

Cell culture and related procedures. Primary human brain microvascular pericyte cells (Applied Cell Biology Research Institute) were grown in Dulbecco's modified Eagle's medium (DMEM); RAW 264.7 cells (TIB-71) and BV2 cells in DMEM medium; HUVECs in M199 medium supplemented with $20 \%$ FBS (Invitrogen), and maintained in an incubator with a humidified atmosphere of $95 \% \mathrm{O}_{2}$ and $5 \% \mathrm{CO}_{2}$ at $37^{\circ} \mathrm{C}$. Raw and $\mathrm{BV} 2$ cells were transfected using Lipofectamine Plus reagent (Invitrogen) for DNA or TransIT-TKO Transfection Reagent (Mirus Bio) for si-RNA. Cell lysates were produced as described previously. ${ }^{39}$ For preparation of conditioned medium (CM) for treating human pericytes/endothelial cells, medium from transfected macrophages was changed into DMEM medium containing $0 \% \mathrm{FBS}$ for $24 \mathrm{~h}$, then collected and filtered through a $0.22-\mu \mathrm{m}$ pore size membrane (Millipore), and then concentrated 10 times using centrifugal filters (Millipore). For CM treatment, cells were grown to $60-70 \%$ confluency, serum starved overnight and stimulated with $500-1000 \mathrm{ng} / \mathrm{ml}$ recombinant human Ang1, Ang2 (R\&D) alone or with $C M$. For signaling experiment, cells were transfected with mock or Ninj1 DNA and then serum starved overnight. SB203580 (Sigma), a p38 inhibitor, was treated for $24 \mathrm{~h}$.

Expression vector construction and western blot analysis. Fulllength wild-type mouse Ninjurin1 (NM_013610) cDNA was produced by RT-PCR from NIH-3T3 fibroblasts. Primers used were: $5^{\prime}$-GGGAATTCCATGGAGT CGGGCACTGAGGA-3' (forward includes enzyme digestion sites for EcoRI) and $5^{\prime}$-CTCCTCGAGTTCTACTGCCGGGGCGCCAC-GT-3' (reverse; for Xhol). The product was inserted into pCS2 + vector (Myc-tagged) for expression in mammalian cells. Transfected macrophages or conditioned media-treated human endothelial cells were analyzed by western blotting. Western blot analysis was performed as described previously. ${ }^{39}$ We used antibodies specific for Ninj1 (BD Pharmingen), c-Myc (Santa Cruz), $\alpha$-tubulin (BioGenex), and caspase-3 antibody (Cell Signaling). Ponceau S solution was purchased from Sigma.

Immunofluorescence microscopy and antibodies. Antibodies used for immunostaining were Ninj1 (1:500, a thankful gift from Dr. J Milbrandt), F4/80 (1:500; Serotec), VE-cadherin (1:500; Santa Cruz), conA (1:1000; Vector Laboratories), GS-lectin (1:1000; Molecular Probes), Iba-1 (1:500; Wako or Abcam), NG2 (1:300; Chemicon), and cleaved-caspase3 antibody (1:500; Cell Signaling). The specificity for Ninj1 antibody was confirmed by several published papers. ${ }^{18,19}$ Apoptosis was detected using Terminal Transferase and Biotin-16dUTP (TUNEL staining; Roche). Nuclei were stained using 4'-6-diamidino-2phenylindole (DAPI; Invitrogen) or propidium iodide (Molecular Probes). Tissues or cells were incubated with the indicated primary antibodies, followed by Alexa488conjugated IgG or Alexa546-conjugated IgG (Molecular Probes) as secondary antibodies. Images were obtained with an Axiovert M200 microscope (Zeiss) and analyzed with image analysis software (NIH-Image J). Vascular density was determined by measuring the area of vessels within eye specimens that were positively immunostained by endothelium-specific GS-lectin antibody through image analysis software (NIH-Image J).

Systemic Ninj1 blockade. ICR mice were assigned to receive intraperitoneal injections of $1 \mathrm{mg} / \mathrm{kg}$ of a mouse-neutralizing antibody to Ninj1 (BD) or $1 \mathrm{mg} / \mathrm{kg}$ of a mouse isotype control antibody (Santa Cruz) at P1. Animals were killed at P6 or P11. Vessels and macrophages in eye were stained with GS-lectin.

Induction of endotoxin shock. SD Rats were injected three times intraperitoneally (IP) with a dose of $100 \mu \mathrm{g}$ of LPS ( $330 \mu \mathrm{g} / \mathrm{kg}$ of body weight) during 3 days. Animals were killed at $24 \mathrm{~h}$ after LPS administration. For in vitro assay, BV2 macrophage cell was treated for $24 \mathrm{~h}$ with 1.0 and $2.5 \mu \mathrm{g} / \mathrm{ml}$ of LPS.

Cell adhesion assays. Adhesion assays were performed using murine BV2 cells transfected with pCS2 +-Ninj1 or pCS2 +-Mock (control) DNA. In cell-cell adhesion assay, BV2 cells were stained with Hoechst (H33342) for $10 \mathrm{~min}$ and washed with DMEM. Then, cells were detached using trypsin/EDTA and detached cells were added into monolayer of mouse brain microvascular endothelial cell with neutralizing antibody in a black flat-bottomed 96-well microtiter plate. Then, plate was incubated for $10 \mathrm{~min}$ and washed with PBS three times. Washed BV2 cells and adhered BV2 cell were collected separately. After lysis with $0.2 \%$ NP- 40 , fluorescence of lysates was analyzed with ELISA at $340 \mathrm{~nm}$. In cell-matrix adhesion assay, BV2 cells were added to each well coated with ECMs, which are fibronectin (Invitrogen), type I/IV collagen (BD), and gelatin (Sigma). After incubation for $15 \mathrm{~min}, \mathrm{BV} 2$ cells were washed with PBS twice. Attached cells were stained with Crystal violet and washed twice. After lysis with $0.2 \%$ NP-40, absorbance of lysates was analyzed with ELISA at $590 \mathrm{~nm}$. Finally, the aggregation formation was examined using a mixture of Ninj1 transfectants and nontransfected cells as previously described. ${ }^{18}$

RT-PCR and real-time PCR. RNA from dissected eyeball preparations or cultured cells was purified using Trizol reagent (Invitrogen). RT-PCR analysis was performed as described previously. ${ }^{39}$ For RT-PCR, the following primers (shown $5^{\prime}$ to $3^{\prime}$ ) were used: gapdh: forward (F), ACCACAGTCCATGCCATCAC; reverse (R), TCCACCACCCTGTTGCTGTA. Ninj1: F, GAGTCGGGCACTGAGGA; R, GTT GCAGGGGTCTGGTCA. Ang1: F, AGGCTTGGTTTCTCGTCAGA; R, TCTGCA CAGTCTCGAAATGG. Ang2: F, GCTGCTGGTTTATTACTGAAGAA; R, TCAGGTG GACTGGGATGTTTAG. Wnt7b: F, AAGAACTCCGAGTAGGGAGTCG; R, TGCGT TGTACTTCTCCTTGAGC. Wnt7b: 2nd round F, CCGAGTAGGGAGTCGAGAGG; R, CACACCGTGACACTTACATTCC. PCR products were analyzed on a $1.2 \%$ agarose gel with ethidium bromide and the gels were digitally imaged. For real-time PCR, the following primers (shown $5^{\prime}$ to $3^{\prime}$ ) were used: Ang2: F, TGTGAT CTTGTCTTGGCCGC; R, AGAGGGAGTGTTCCAAGAAGC. Gapdh, Ang1, and Wnt7b were done with the same primers of RT-PCR. Real-time PCR was performed on the LightCycler (LC) 2.0 instrument (Roche) using Lightcycler FastStart DNA Master SYBR Green I (Roche) as the manufacturer's instructions.

Small interfering RNAs (siRNAs) knockdown. Ninj1 knockdown was performed with IDT TriFECTA dicer substrate (DsiRNA) duplexes that were designed to inhibit expression of Ninj1 in mouse cells using TriFECTa Dicer-Substrate RNAi system (IDT Inc.). Three different siRNAs against mouse Ninj1 (NM_013610) were designed using IDT RNAi Design Software and purchased from IDT. Briefly, BV2 and Raw264.7 cells were plated at a density of $2 \times 10^{4}$ cells per well in a 24-well plate. After $24 \mathrm{~h}$, cells were transfected using 20,10 , or $1 \mathrm{nM}$ siRNA duplexes. Hypoxanthine guanine phosphoribosyltransferase (HPRT)-S1 positive control and scrambled negative control (sc) duplexes were used at concentration of 20 or $10 \mathrm{nM}$. TransIT-TKO Transfection Reagent (Mirus Bio) was used for all transfections. Optimal inhibition of Ninj1 expression was achieved by using transfection of $20 \mathrm{nM}$ Ninj1-siRNA. Cells were analyzed for Ninj1 and Wnt7b expression by RT-PCR after $24 \mathrm{~h}$ of transfection.

Data analysis and statistics. Quantification of band intensity was analyzed using ImageJ (http://rsb.info.nih.gov/ij/) and normalized to the density of the gapdh, tubulin, or ponceau $S$ staining band. All data are presented as mean \pm S.D. and changed into relative percentage. Statistical comparisons between groups were done using Student's $t$-test. $P<0.05$ was considered statistically significant.

Acknowledgements. We thank Dr. Jeffrey Milbrandt (University of Washington, USA) and Dr. Toshiyuki Araki (National Institute of Neuroscience, 
Japan) for providing the Ninj1 antibody. We also thank Dr. Mi In Roh (Yonsei University, Korea) for linguistic corrections. This work was supported by the Korea Science and Engineering Foundation (KOSEF) grant funded by the Korea government (MEST) through the Creative Research Initiative program (Grant R162004-001010010-2008).

Author contributions: H-JL planned the project, performed the experiments, analyzed the data, and wrote the article; BJA and MWS performed the experiments and analyzed the data; J-WJ contributed to the data analysis and to the writing of the article; JHK provided advice to the data analysis on the ocular experiment; $\mathrm{K}$ WK planned the project, analyzed the data, and wrote the article.

\section{Conflict of interest. The authors declare no conflict of interest.}

1. Chambon JP, Soule J, Pomies P, Fort P, Sahuquet A, Alexandre D et al. Tail regression in Ciona intestinalis (Prochordate) involves a Caspase-dependent apoptosis event associated with ERK activation. Development 2002; 129: 3105-3114.

2. Montero JA, Ganan Y, Macias D, Rodriguez-Leon J, Sanz-Ezquerro JJ, Merino R et al. Role of FGFs in the control of programmed cell death during limb development. Development 2001; 128: 2075-2084.

3. Cowan WM, Fawcett JW, O'Leary DD, Stanfield BB. Regressive events in neurogenesis. Science 1984; 225: 1258-1265.

4. Saint-Geniez M, D'Amore PA. Development and pathology of the hyaloid, choroidal and retinal vasculature. Int J Dev Biol 2004; 48: 1045-1058.

5. Hurskainen M, Eklund L, Hagg PO, Fruttiger M, Sormunen R, Ilves M et al. Abnormal maturation of the retinal vasculature in type XVIII collagen/endostatin deficient mice and changes in retinal glial cells due to lack of collagen types XV and XVIII. FASEB J 2005; 19 : 1564-1566.

6. Rao S, Lobov IB, Vallance JE, Tsujikawa K, Shiojima I, Akunuru S et al. Obligatory participation of macrophages in an angiopoietin 2-mediated cell death switch. Development 2007; 134: 4449-4458.

7. Lobov IB, Rao S, Carroll TJ, Vallance JE, Ito M, Ondr JK et al. WNT7b mediates macrophage-induced programmed cell death in patterning of the vasculature. Nature 2005 437: 417-421.

8. Reichel MB, Ali RR, D'Esposito F, Clarke AR, Luthert PJ, Bhattacharya SS et al. High frequency of persistent hyperplastic primary vitreous and cataracts in p53-deficient mice. Cell Death Differ 1998; 5: 156-162.

9. Lang RA, Bishop JM. Macrophages are required for cell death and tissue remodeling in the developing mouse eye. Cell 1993; 74: 453-462.

10. Diez-Roux G, Argilla M, Makarenkova H, Ko K, Lang RA. Macrophages kill capillary cells in G1 phase of the cell cycle during programmed vascular regression. Development 1999; 126: 2141-2147.

11. Diez-Roux G, Lang RA. Macrophages induce apoptosis in normal cells in vivo. Development 1997; 124: 3633-3638.

12. Chang B, Smith RS, Peters M, Savinova OV, Hawes NL, Zabaleta A et al. Haploinsufficient Bmp4 ocular phenotypes include anterior segment dysgenesis with elevated intraocular pressure. BMC Genet 2001; 2: 18

13. Willert K, Brown JD, Danenberg E, Duncan AW, Weissman IL, Reya T et al. Wnt proteins are lipid-modified and can act as stem cell growth factors. Nature 2003; 423: 448-452.

14. Mitchell CA, Risau W, Drexler HC. Regression of vessels in the tunica vasculosa lentis is initiated by coordinated endothelial apoptosis: a role for vascular endothelial growth factor as a survival factor for endothelium. Dev Dyn 1998; 213: 322-333.

15. Rutland CS, Mitchell CA, Nasir M, Konerding MA, Drexler HC. Microphthalmia, persistent hyperplastic hyaloid vasculature and lens anomalies following overexpression of VEGFA188 from the alphaA-crystallin promoter. Mol Vis 2007; 13: 47-56.

16. Shui YB, Wang X, Hu JS, Wang SP, Garcia CM, Potts JD et al. Vascular endothelial growth factor expression and signaling in the lens. Invest Ophthalmol Vis Sci 2003; 44 3911-3919.
17. Alvarez Y, Cederlund ML, Cottell DC, Bill BR, Ekker SC, Torres-Vazquez J et al. Genetic determinants of hyaloid and retinal vasculature in zebrafish. BMC Dev Biol 2007; 7: 114.

18. Araki T, Milbrandt J. Ninjurin, a novel adhesion molecule, is induced by nerve injury and promotes axonal growth. Neuron 1996; 17: 353-361.

19. Araki T, Zimonjic DB, Popescu NC, Milbrandt J. Mechanism of homophilic binding mediated by ninjurin, a novel widely expressed adhesion molecule. J Biol Chem 1997; 272: 21373-21380.

20. Araki T, Milbrandt J. Ninjurin2, a novel homophilic adhesion molecule, is expressed in mature sensory and enteric neurons and promotes neurite outgrowth. J Neurosci 2000; 20: 187-195.

21. Schlosshauer B, Schwarz U, Rutishauser U. Topological distribution of different forms of neural cell adhesion molecule in the developing chick visual system. Nature 1984; 310 : 141-143.

22. Jakovcevski I, Wu J, Karl N, Leshchyns'ka I, Sytnyk V, Chen J et al. Glial scar expression of $\mathrm{CHL} 1$, the close homolog of the adhesion molecule L1, limits recovery after spinal cord injury. J Neurosci 2007; 27: 7222-7233.

23. Kim JW, Moon AR, Kim JH, Yoon SY, Oh GT, Choe YK et al. Up-Regulation of ninjurin expression in human hepatocellular carcinoma associated with cirrhosis and chronic viral hepatitis. Mol Cells 2001; 11: 151-157.

24. Zhang S, Dailey GM, Kwan E, Glasheen BM, Sroga GE, Page-McCaw A. An MMP liberates the Ninjurin A ectodomain to signal a loss of cell adhesion. Genes Dev 2006; 20: 1899-1910.

25. Brown AS, Leamen L, Cucevic V, Foster FS. Quantitation of hemodynamic function during developmental vascular regression in the mouse eye. Invest Ophthalmol Vis Sci 2005; 46: 2231-2237.

26. Zhu M, Madigan MC, van Driel D, Maslim J, Billson FA, Provis JM et al. The human hyaloid system: cell death and vascular regression. Exp Eye Res 2000; 70: 767-776.

27. Meeson A, Palmer M, Calfon M, Lang R. A relationship between apoptosis and flow during programmed capillary regression is revealed by vital analysis. Development 1996; 122: 3929-3938.

28. Yu WM, Yu H, Chen ZL, Strickland S. Disruption of laminin in the peripheral nervous system impedes nonmyelinating Schwann cell development and impairs nociceptive sensory function. Glia 2009; 57: 850-859.

29. De Angelis E, MacFarlane J, Du JS, Yeo G, Hicks R, Rathjen FG et al. Pathological missense mutations of neural cell adhesion molecule L1 affect homophilic and heterophilic binding activities. EMBO J 1999; 18: 4744-4753.

30. Mauro VP, Krushel LA, Cunningham BA, Edelman GM. Homophilic and heterophilic binding activities of Nr-CAM, a nervous system cell adhesion molecule. J Cell Biol 1992; 119: 191-202.

31. Rosso SB, Sussman D, Wynshaw-Boris A, Salinas PC. Wnt signaling through Dishevelled, Rac and JNK regulates dendritic development. Nat Neurosci 2005; 8: 34-42.

32. Affolter M, Bellusci S, Itoh N, Shilo B, Thiery JP, Werb Z. Tube or not tube: remodeling epithelial tissues by branching morphogenesis. Dev Cell 2003; 4: 11-18.

33. Zhan Y, Brown C, Maynard E, Anshelevich A, Ni W, Ho IC et al. Ets-1 is a critical regulator of Ang II-mediated vascular inflammation and remodeling. J Clin Invest 2005; 115: 2508-2516.

34. Liang CP, Han S, Senokuchi T, Tall AR. The macrophage at the crossroads of insulin resistance and atherosclerosis. Circ Res 2007; 100: 1546-1555.

35. Sunderkotter C, Steinbrink K, Goebeler M, Bhardwaj R, Sorg C. Macrophages and angiogenesis. J Leukoc Biol 1994; 55: 410-422.

36. Ambili M, Jayasree K, Sudhakaran PR. 60K gelatinase involved in mammary gland involution is regulated by beta-oestradiol. Biochim Biophys Acta 1998; 1403: 219-231.

37. Santos AM, Calvente R, Tassi M, Carrasco MC, Martin-Oliva D, Marin-Teva JL et al. Embryonic and postnatal development of microglial cells in the mouse retina. J Comp Neurol 2008; 506: 224-239.

38. Remington LT, Babcock AA, Zehntner SP, Owens T. Microglial recruitment, activation, and proliferation in response to primary demyelination. Am J Pathol 2007; 170: 1713-1724.

39. Jeong $\mathrm{CH}$, Lee HJ, Cha JH, Kim JH, Kim KR, Kim JH et al. Hypoxia-inducible factor-1 alpha inhibits self-renewal of mouse embryonic stem cells in vitro via negative regulation of the leukemia inhibitory factor-STAT3 pathway. J Biol Chem 2007; 282: 13672-13679.

Supplementary Information accompanies the paper on Cell Death and Differentiation website (http://www.nature.com/cdd) 